\title{
Organosilane modified Zr-based conversion layer on Zn-Al alloy coated steel sheets
}

\author{
Thomas Lostak, ${ }^{{ }^{*}}$ Christian Timma, ${ }^{\ddagger}$ Stefan Krebs, ${ }^{\ddagger}$ Jörg Flock,,${ }^{\ddagger}$ Stephan Schulz ${ }^{\dagger}$ \\ thyssenkrupp Steel Europe AG, Research and Development, Kaiser-Wilhelm-Straße 100, 47166 Duisburg, Germany \\ †Universität Duisburg-Essen, Faculty of Chemistry, Universitätsstraße 7, 45141 Essen, Germany
}

\begin{abstract}
Zirconium based conversion layers are deliberated as environmentally friendly alternatives replacing classical trication phosphatation and chromate-based passivation layers in the automotive and coil-coating industry. Based on excellent electronic barrier properties they provide an effective corrosion protection of the metallic substrate. To investigate the influence of organofunctional silanes on the protection properties of these pre-treatment systems, 3gylcidoxypropyltrimethoxysilane ( $\gamma$-GPS) was added to the aqueous model conversion solution containing well-defined amounts of hexafluorozirconic acid $\left(\mathrm{H}_{2} \mathrm{ZrF}_{6}\right)$. The surface composition of these modified conversion layers were determined using X-ray photoelectron spectroscopy (XPS), infrared reflection absorption spectroscopy (FT-IRRAS) and confocal Raman microscopy (CRM). Scanning Kelvin Probe (SKP) measurements were obtained on organic coated samples in order to investigate the corrosive cathodic delamination process in more detail.
\end{abstract}

Keywords: $\mathrm{ZrO}_{2}$, zinc alloy coated steel sheets, conversion layer, organofunctional silanes, corrosion protection

\footnotetext{
${ }^{*}$ Corresponding author

Email address: thomas.lostak@thyssenkrupp.com

Phone: +49 203 52-244521

Fax: +49 203 52-228723
} 


\section{Introduction}

Well-established pre-treatment systems for metal substrates based on chromates [1-3] and phosphates [4,5] are used to provide excellent adhesion properties for organic coatings and improve the corrosion resistance of the underlying metal substrates. However, there are serious disadvantages of these pre-treatment systems such as their inefficient process parameters, their grave ecological impact and their unacceptable toxicity [6]. As a consequence, the use of hexavalent chromate passivation $(\mathrm{Cr}(\mathrm{VI}))$ has been restricted and internationally banned (e.g. REACH, RoHS) [7, 8]. Recently, new environmentally acceptable pre-treatment systems were developed. In particular zirconium based conversion coatings are promising alternatives to substitute detrimental pre-treatment technologies in the field of metal finishing [9-12]. In the coil-coating and automotive industry predominantly hexafluorozirconic acid $\left(\mathrm{H}_{2} \mathrm{ZrF}_{6}\right)$ [13-25] is used to form the conversion coating on the metal substrate. Different studies showed that the applied conversion coating mostly consists of $\mathrm{ZrO}_{2}$, fluorine and small quantities of the metallic substrate $[22,23,25,26]$.

The formation mechanism of Zr-based protection layers was elucidated for different metallic substrates $[13,16,21,25,26]$. The precipitation of the conversion layer generally requires an anodic dissolution of the metallic substrate and a subsequent cathodic alkalization of the electrolyte in close proximity to the sample surface. Recent studies reported that the formation of the ultra-thin conversion layers, which typically range from 5 to $150 \mathrm{~nm}$, highly depends on the microstructure of the alloy composition, which mainly determines the electrochemical behaviour of the metal surfaces $[18,26,27]$. Nordlien et al. showed that the preferred deposition of the $\mathrm{ZrO}_{2}$ layer on aluminium alloys occurred on and around intermetallic (nano)particles (IMP) due to specific cathodic reactions such as hydrogen evolution and oxygen reduction, resulting in a reduced activity of the IMPs due to $\mathrm{ZrO}_{2}$ precipitation [15]. The formation mechanism of a protective Zr-based conversion layer on a steel substrate 
coated with a thin layer of a Zn-Al-Mg alloy was demonstrated by Lostak et al. [26]. They used a set of sophisticated EDX, AFM and SKPFM experiments to show that the $\mathrm{ZrO}_{2}$ deposition starts on Zn-rich phases (local cathodes). With longer immersion times the precipitation of the conversion layer starts on Mg-rich phases and covers subsequently the complete surface.

Various electrochemical investigations (electrochemical impedance spectroscopy measurements, in situ height-regulated scanning Kelvin probe studies [22], potentiodynamic polarization experiments [25]) clearly showed superior corrosion protection properties of zirconium based conversion layers on a wide range of alloys [22-26]. In addition, decreased kinetics of the cathodic delamination of organic coatings applied on $\mathrm{Zn}$-Al alloy surfaces were observed [22]. However, this pre-treatment technology will not replace the established phosphating systems in the automotive industry as long as the requested performance is missed. Promising approaches are the combination of inorganic conversion coatings with an adhesion-promoting layer [28-31]. Different authors showed that the corrosive delamination process can significantly be reduced due to the use of appropriate coupling reagents. Recent studies show that the corrosion protection of such systems can be improved if a composite is deposited on the metallic substrate prior to lacquers process. The concept behind such an interface modification is the combination of an insulating layer (usually a conversion coating), in the sense of an electronic transfer barrier, with a suitable adhesion promoter for a covalent bond to the following organic coating. Schinkinger et al. deposited ultrathin nanoscaled silica like layers using combustion chemical vapour deposition (CCVD) on zinc coated steel as an interface layer between the metallic substrate and the following organic coating $[29,30]$. The barrier properties and coverage of these thin $\mathrm{SiO}_{2}$-layers were proven by atomic force microscopy (AFM) and cyclic voltammetry (CV), demonstrating that $99 \%$ coverage of the substrate can be achieved in very short deposition times. The authors modified the interface with an ultra-thin 3-glycidoxypropyltrimethoxysilane ( $\gamma$-GPS) adhesion 
1 promoting film $(5-10 \mathrm{~nm})$ to achieve the performance of a chromate conversion coating. The

2 surface analytical characterization of the applied films was performed with infra-red reflection

3 absorption spectroscopy (FT-IRRAS) and X-ray photoelectron spectroscopy (XPS). A similar

4 approach was followed by Wapner et al. [28,31]. They modified the adhesive / metal interface

5 with two different kinds of ultra-thin layers:

6 (1) $\mathrm{HMDS} / \mathrm{O}_{2} \mathrm{SiO}_{\mathrm{x}}$-like plasma polymer layers to inhibit the electron transfer at the interface

7 to prevent the oxygen reduction reaction and

8 (2) 3-(trimethoxysilyl)-propylamine ( $\gamma$-APS) layer to form a stable covalent bond to the epoxy

9 resin adhesive.

The authors proved the very good corrosion protection performance of such systems by using electrochemical methods (in situ height-regulated scanning kelvin probe studies, potentiodynamic polarization experiments).

In this study, the influence of organofunctional silanes on the protection properties of ultrathin Zr-based conversion layers is investigated in detail. For this purpose, hot dipped galvanized steel sheets (HDG) were treated in an aqueous model conversion solution containing well-defined amounts of hexafluorozirconic acid. 3glycidoxypropyltrimethoxysilane $(\gamma$-GPS) was chosen as coupling agent. Two different application processes were compared: one coating step procedure (only one coating solution containing $\mathrm{H}_{2} \mathrm{ZrF}_{6}$ and $\gamma$-GPS - denoted as VBH-E) and two coating step procedure (subsequent two different coating solutions, $\mathrm{H}_{2} \mathrm{ZrF}_{6}$ and $\gamma$-GPS - denoted as VBH-S). The surface of the resulting conversion coatings was investigated by X-ray photoelectron spectroscopy (XPS), infrared reflection absorption spectroscopy (FT-IRRAS) and confocal Raman microscopy (CRM). Scanning Kelvin Probe (SKP) measurements were performed on 
1 organic coated specimens in order to characterize the corrosive cathodic delamination 2 process.

3 
1

2

3 Samples of line produced skin-passed hot dip galvanized steel blanks with a diameter of $5 \mathrm{~cm}$

4 (ThyssenKrupp Steel Europe AG) were used. The galvanized layer contains 99,84 \% Zn and 5

\section{Experimental}

\subsection{Materials and sample preparation}

$1.16 \% \mathrm{Al}$ by weight. To ensure reproducible clean surfaces, all samples were degreased using an automated cleaning system (WESERO GmbH, Germany). The cleaning procedure contains following steps:

Step 1 - Ridoline ${ }^{\circledR} \mathrm{C} 72$ (Henkel KGaA, Germany), $8 \mathrm{~g} / 1,70 \pm 5^{\circ} \mathrm{C}, \mathrm{pH} 11$ (cleaning time: $8 \mathrm{~s}$ )

Step 2 - Ridoline ${ }^{\circledR} 1340$ (Henkel KGaA, Germany), 16-20 g/l, $70 \pm 5{ }^{\circ} \mathrm{C}, \mathrm{pH} 9.9$ (cleaning time: $8 \mathrm{~s}$ )

Step 3 - Rinsing with deionised water $\left(50{ }^{\circ} \mathrm{C}, \kappa=\leq 4 \mu \mathrm{S} / \mathrm{cm}\right)$

Step 4 - Drying in warm stream of air

\subsection{Conversion layer deposition}

The deposition of the conversion layer by using a conversion solution which contains 1 $\mathrm{mmol} / \mathrm{l}$ hexafluorozirconic acid $\left(\mathrm{H}_{2} \mathrm{ZrF}_{6}\right)$ solution was performed directly after degreasing of the substrates. The $\mathrm{pH}$ value of the conversion solution was adjusted to $\mathrm{pH} 4$ using ammonium bicarbonate $\left(\mathrm{NH}_{4} \mathrm{HCO}_{3}\right)$ as buffer agent. The $\gamma$-GPS solution was prepared from an aqueous solution ( $\mathrm{pH} 4$, adjusted with acetic acid) with 1 wt. $\% \gamma$-GPS. Prior to the coating procedure, the solution was stirred for 3 hours to complete the hydrolysis reaction. Immersion of the samples was performed under solution stirring $(300 \mathrm{rpm})$ at ambient temperature $\left(T \sim 20^{\circ} \mathrm{C}\right)$. After finishing the pre-treatment procedure, all substrates were rinsed with ultrapure water $\left(T \sim 20^{\circ} \mathrm{C}, \kappa \leq 0,005 \mu \mathrm{S} / \mathrm{cm}\right)$ and dried in a stream of nitrogen gas. Subsequent, the silane films were cross-linked for 15 minutes at $100^{\circ} \mathrm{C}$. The composition of 
1 the different conversion solutions and the used application parameters are summarized in

2 table 1.

4 For the investigation of cathodic delamination, $50 \%$ of the surfaces of alkaline cleaned

\subsection{Preparation of organic coated samples for HR-SKP measurements}

samples were masked with an adhesive tape before immersing in the different pre-treatment solutions. After the pre-treatment procedure (see 2.2) the uncoated zinc surface was uncovered again by pulling off the adhesive tape. Afterwards, a commercial two component lacquer (Glasurit $\AA^{\circledR}$ ) with an isocyanate hardener (MS-Clear 923-155, BASF Coatings AG, Germany) was applied by spin coating (Primus STT115, Fa. ATM Vision, Germany) at 4500 rpm for $10 \mathrm{~s}$ producing a film thickness of about $4-5 \mu \mathrm{m}$ on the hot dipped galvanized steel sheets. Subsequent, the polymer films were cross-linked for 20 minutes at $60{ }^{\circ} \mathrm{C}$. Lastly, an 5 wt.\% $\mathrm{NaCl}$ electrolyte was added to the uncoated defect area to start the cathodic delamination process. This specific sample preparation made it possible to investigate two different interfaces in a single HR-SKP measurement. The used preparation procedure was adapted from Wapner and Grundmeier [28] and is more detailed described in an essay from Klimow et al. [32].

\subsection{Surface analytical techniques}

\subsubsection{FT-IRRAS}

All obtained infrared reflection absorption spectra of the applied conversion layers were measured using a FTS 3000 Spectrometer (Digilab, Germany). The spectrometer was constantly purged with dried nitrogen and equipped with a DTGS (deuterated triglycine sulphate) detector. All spectra were recorded using a resolution of $4 \mathrm{~cm}^{-1}$ and originate from co-addition of 512 single scans. The mid infrared beam is guided by a set of mirrors and transmitted through $\mathrm{ZnSe}$ window onto the sample and reflected under $80^{\circ}$ to the detector. 
2 Raman spectroscopic studies were carried out on an Alpha 300 R confocal Raman microscope

(WITec GmbH, Germany). All spectra were recorded with a $488 \mathrm{~nm} \mathrm{Ar}^{+}$-laser (Spectra Physics, USA) as excitation source on vertical polarization (CCD-Detector). The laser power was set at $32 \mathrm{~mW}$. The exposure time was between 10-20 s for each spectrum to inhibit any damage of the used laser to the sample. If not otherwise indicated, IR spectra were recorded through a 600 lines/mm grating. The imaging of the collected scans was performed through WiTec Project® (Version 2.10).

\subsubsection{XPS}

The chemical compositions of the conversion layers were determined using a Quantum 2000 ESCA Microprobe (Physical Electronics Inc., USA). All spectra were obtained using a monochromated $\mathrm{Al} \mathrm{K}_{\alpha}$-beam with a spot size of $100 \times 100 \mu \mathrm{m}$. The take-off angle of the detected photoelectrons was $45^{\circ}$ to the surface normal. The $\mathrm{C} 1 \mathrm{~s}$ peak (binding energy of $284.8 \mathrm{eV}$ ) was used as internal reference for all spectra. The quantification of all elements was performed on the basis of survey spectra. Depth profiling was performed using $\mathrm{Ar}^{+}$ion sputtering with an acceleration voltage of $2 \mathrm{kV}$ and sputter rate of $8.5 \mathrm{~nm} / \mathrm{min}$. A silicon wafer containing a well-defined oxide layer of known thickness was used for depth calibration. The fitting of the measured XPS spectra was performed through the CasaXPS software 2.3.15 (Casa Software).

\subsection{HR-SKP measurements}

The cathodic delamination experiments were performed with a height regulated Scanning Kelvin Probe (SoftControl, Germany). Prior to HR-SKP experiments organically coated samples were pre-exposed in humid atmosphere to remove any electrostatic charging of the surface. A steady gas flow rate in the SKP chamber was kept during the measurements to 
1

2

ensure a constant atmosphere with a defined relative humidity ( $>90 \%$ r.H.). A NiCr needle with a tip diameter of $100 \mu \mathrm{m}$ was used for all measurements. The calibration of the $\mathrm{NiCr}$ needle was accomplished by measuring and referring to a known $\mathrm{Cu} / \mathrm{CuSO}_{4}$ electrode. All potentials measured with the Scanning Kelvin Probe are with respect to the Standard Hydrogen Electrode (SHE). For statistical reasons each measurement was repeated three times.

\section{Results and discussion}

\subsection{Surface analytical studies of silane-modified conversion coatings}

X-ray photoelectron spectroscopy was applied to investigate the general composition of the different conversion layers. The concentration of the chemical elements on the surface of the conversion layer is summarized in table 2 . These studies confirmed the typical composition of these layers, which consist of small quantities of metallic zinc $(\sim 5-11$ at.\%), fluorine $\quad(\sim 2-$ 3 at.\%), zirconium ( $\sim 5-16$ at.\%) and oxygen $(\sim 50-55$ at.\%) $[25,26]$. The silicon $(\sim 5-9$ at.\%) and carbon $(\sim 17-22$ at. $\%)$ content results from the $\gamma$-GPS in the conversion coating. Moreover, it cannot be excluded that a certain proportion of the carbon and oxygen content originate from oxygen containing organic adsorbents present at the conversion coating surface. The associated XPS survey spectra of the different conversion layer are shown in figure 1 . The different levels of the various elements indicate a varying layer structure of the two different application procedures.

To obtain a deeper insight into the layer structure of the different pre-treatment systems, XPS depth profiles were recorded. Fig. 2 shows the XPS sputter profile of the sequentially coated sample (VBH-S). As expected $[25,26]$, the thickness of the applied layers is in the nanometer range. Nonetheless, the trend of the Zr3d- and Si2p-signal clearly shows the defined layer structure of the conversion coating. While the Si2p-signal drops to about 3-5 nm, the $\mathrm{Zr} 3 \mathrm{~d}$ 
signal reaches its maximum between $4-5 \mathrm{~nm}$ and then decreases gradually, while the $\mathrm{Zn} 2 \mathrm{p} 3$ signal begins to rise from the metallic zinc coating. The sputter profile of the one step coated specimen is presented in fig. 3 . In contrast to the sequentially coated sample, no defined layer structure was observed. Both the Zr3d- and Si2p-signal show a parallel course over the whole layer. To obtain more accurate information about the molecular structure of the adherent conversion coatings, IRRAS and Raman imaging measurements were performed.

The infrared spectra of the both samples are shown in fig. 4. Both spectra show bands of medium-to-strong intensity between $1200-800 \mathrm{~cm}^{-1}$. The line at $1113 \mathrm{~cm}^{-1}$ can be assigned to the Si-O-Si deformation vibration, which is caused by the hydrolysis and reaction of $\gamma$-GPS on the steel surface. These values agree with vibration bands reported by Grundmeier et al. ( $1162 \mathrm{~cm}^{-1}$ ) [45]. The vibration band at $923 \mathrm{~cm}^{-1}$ can be assigned to free $\mathrm{Si}-\mathrm{OH}$ groups, proving the presence of unreacted molecules on the sample surface. That is further confirmed by the presence of C-H stretching vibrations of $\mathrm{CH}_{3}$ groups between $3000-2800 \mathrm{~cm}^{-1}$, which are still bound to the used siloxane. Si-O-Zr vibrations, that indicate the reaction and linking of $\gamma$-GPS to $\mathrm{ZrO}_{2}$ on the surface, are supposed to appear in the range of $980-930 \mathrm{~cm}^{-1}$. Ivanova et al. reported similar bands at $980 \mathrm{~cm}^{-1}$ corresponding to $\mathrm{Si}-\mathrm{O}-\mathrm{Zr}$ vibrations [34]. Due to the broad band assigned to the Si-OH stretching vibrations $\left(923 \mathrm{~cm}^{-1}\right)$ this band might be overlapped. A small shoulder found at $860 \mathrm{~cm}-1$ can be assigned to $\mathrm{C}-\mathrm{O}-\mathrm{C}$ vibrations from the epoxy ring adjacent to $\gamma$-GPS [36]. Zr-O vibrations are shown in the low frequency area between $750-400 \mathrm{~cm}^{-1}$.

To summarize, both spectra show a comparable surface layer on the samples, independent of the sample treatment. Both preparation methods show a successful deposition of $\mathrm{ZrO}_{2}$ and $\gamma$ GPS on the surface. Furthermore, the Si-O-Si vibration bands indicate a linkage and reaction of $\gamma$-GPS forming a thin solid film on the steel surface. Otherwise a reaction between the silane molecules and $\mathrm{ZrO}_{2}$ could not be shown and has to be investigated by different 
methods. However, there are significant differences in the intensities of the vibration bands obtained in both spectra. The intensities of vibrations related to $\gamma$-GPS are decreased on sample VBH-S, confirming the XPS measurements showing a lower amount of the silane coupling reagent on this sample. Furthermore, $\mathrm{ZrO}_{2}$ related bands are much more intensive on this surface.

In order to visualize the lateral distribution of $\mathrm{ZrO}_{2}$ and $\gamma$-GPS on the sample surfaces, complementary Raman imaging studies were performed. Fig. 5 shows the recorded Raman spectra of the two different prepared samples.

The spectra generally provide the same information like the infrared spectra. Vibration bands assigned to $\mathrm{ZrO}_{2}$ occur in the spectral region between $650-300 \mathrm{~cm}^{-1}$. The Raman spectrum of sample VBH-S reveals a broad vibration between $700-250 \mathrm{~cm}^{-1}$ with several peaks at 376 , 421, 492 and $547 \mathrm{~cm}^{-1}$ that indicate a $\mathrm{ZrO}_{2}$ film on the surface (s. fig. 5). Such a broad peak was also recorded by Keramidas et al. measuring different polymorphic states of $\mathrm{ZrO}_{2}$ [46]. Depending on the temperature treatment, $\mathrm{ZrO}_{2}$ adopts either a tetragonal $\left(200^{\circ} \mathrm{C}-1000^{\circ} \mathrm{C}\right)$ or a monoclinic (over $1000^{\circ} \mathrm{C}$ ) crystal structure. Since the sample preparation is carried out at ambient temperature, the $\mathrm{ZrO}_{2}$ layer is predominantly amorphous and only a few peaks can be identified in the lower frequency range. The intensity of vibrations assigned to $\gamma$-GPS are low resolved on VBH-S, but peaks according to $\mathrm{C}-\mathrm{H}$ vibrations in the area of $3000-2800 \mathrm{~cm}-1$ and Si-O related vibrations between $1250-1000 \mathrm{~cm}^{-1}$ were detected. On the samples coated in a 1-step process (VBH-E), stronger peaks in the range of $1500-750 \mathrm{~cm}^{-1}$ were observed, which can be attributed to the silane coupling reagent. Bands at $2926 \mathrm{~cm}^{-1}$ and $2893 \mathrm{~cm}^{-1}$ can be assigned to $\mathrm{C}-\mathrm{H}$ vibrations and peaks at $1445 \mathrm{~cm}^{-1}$ and $1300 \mathrm{~cm}^{-1}$ to $\mathrm{C}-\mathrm{C}$ vibrations of the $\gamma$-GPS. A broad band between 1250 and $1000 \mathrm{~cm}^{-1}$ most likely results from Si-O and/or C-C vibrations. 
1 To investigate the deposition behaviour of $\mathrm{ZrO}_{2}$ and $\gamma$-GPS depending on the preparation

2 method, definite area scans on a scanning frame of $10 \times 10 \mu \mathrm{m}$ (100 lines per image, 100

3 points per line) were performed on each sample surface. In a stepwise raster the microscope is recording $100 \times 100$ Raman spectra in the scanning frame that allow a visualization of surface adherent compounds in accordance with the intensity of their vibrational bands. The intensity of the observed bands can be represented in different colour levels, which are calculated from the area under the considered band. Brighter areas in the image correspond to a higher intensity of the observed band while dark areas show lower intensities. A detailed view on this analysis method can be found in the work of Dieing et al. [47].

Microscopic images of the sample surfaces including the performed Raman imaging scans are illustrated in fig. 6. To visualize the reacted $\gamma$-GPS and $\mathrm{ZrO}_{2}$ specific vibration areas of both substances were used. In $\gamma$-GPS the $\mathrm{C}$-H related stretching vibrations cause the most intense bands in the Raman spectra, therefore the range between $3000-2800 \mathrm{~cm}^{-1}$ was used for imaging this substance. For $\mathrm{ZrO}_{2}$ the area of $550-350 \mathrm{~cm}^{-1}$, which includes the related vibrations, was chosen.

The spectra collected on sample VBH-S (s. fig. 6 left) reveal a well distributed film of $\mathrm{ZrO}_{2}$ and $\gamma$-GPS on the surface. A few areas show a higher intensity (=brighter colour) of $\mathrm{ZrO}_{2}$ related vibrations according to the formation of $\mathrm{ZrO}_{2}$ agglomerations. Concerning the intensity of the $\mathrm{C}-\mathrm{H}$ related vibrations of $\gamma$-GPS, the silane film also seems to be homogenously coated onto the $\mathrm{ZrO}_{2}$ coating.

Sample VBH-E shows different deposition behaviours of $\gamma$-GPS and $\mathrm{ZrO}_{2}$. The intensity of $\gamma$ GPS is higher compared to that of the VBH-S sample, but it also shows higher intensity variations in the calculated image. Such variations indicate a worse distribution and less homogenous deposition of $\gamma$-GPS and have also been observed in the Raman images of $\mathrm{ZrO}_{2}$ on this sample. Overall the $\mathrm{ZrO}_{2}$ vibrations have lower intensities (s. fig. 6 right) compared to 
1 the bands obtained on VBH-S. Dark zones in the image of $\mathrm{ZrO}_{2}$ show less coated areas, but

2 actually reveal a high intensity of $\gamma$-GPS related bands as seen by the corresponding image of $3 \gamma$-GPS.

4 Summarizing, the preparation method was found to have a significant influence on the surface 5 layer characteristics of the resulting samples. Surfaces coated in a 2-step process reveal a more homogenous distribution of $\mathrm{ZrO}_{2}$ and $\gamma$-GPS than in 1-step processes, which most likely results from the batched preparation method. At first the sample was immersed in a $\mathrm{H}_{2} \mathrm{ZrF}_{6}$ solution to form a $\mathrm{ZrO}_{2}$ layer and after that in a $\gamma$-GPS solution. Actually there is no competitive reaction between the silane molecules and $\mathrm{H}_{2} \mathrm{ZrF}_{6}$ since $\mathrm{ZrO}_{2}$ is firstly coated onto the surface. This results in homogenously distributed films in both preparation steps.

The Raman images of sample VBH-E show, that a competitive reaction between $\mathrm{H}_{2} \mathrm{ZrF}_{6}, \gamma$ GPS and the sample surface occurs when using a 1-step preparation method with both substances simultaneously. This leads to primarily $\mathrm{ZrO}_{2}$ or $\gamma$-GPS coated areas.

\subsection{HR-SKP investigations of cathodic delamination on multilayer samples}

The height-regulated Kelvin probe is a well-established space-resolved method for investigating the barrier properties of conversion layers or pre-treatments, organic coatings and multilayer films $[3,22,28,32]$. The delamination mechanism of organic coatings consists of two part reactions. These are locally separated, namely the cathodic oxygen reduction (ORR, local cathode) and - in the case of zinc alloy coatings - the anodic zinc dissolution reaction (local anode). The ORR starts for example at a mechanical defect of the organic coating, while the corresponding zinc dissolution occurs on places where the organic coating is completely delaminated. The ORR generates bond breaking reactive radicals and a subsequent alkalisation of the interface between the organic coating, conversion layer and the metallic interface. The loss of adhesion in this interface area finally results in a complete 
1 delamination of the organic coating [32]. The conversion layer stabilizes the interface

2 between the metallic substrate and the organic coating by retarding the electron transfer

3 kinetic of the ORR. A more detailed description of the cathodic delamination mechanism can

4 be found in an essay from Klimow et al. [32].

5 The local cathode of the ORR at the delamination front and the local anode of the zinc dissolution can be detected by space-resolved potential measurements via SKP. The local cathode corresponds to a more positive potential compared to the local anode. Thereby the delamination front of the organic coating is characterized by an s-shaped potential gradient. The time-dependent shift of its point of inflexion is used to evaluate the delamination velocity $\left(v_{D e l}\right)$. By a linear adjustment of the time-dependent front position $v_{\text {Del }}$ of the organic coating can be determined in $\mu \mathrm{m} / \mathrm{h}[22,28]$.

In situ HR-SKP measurements were performed with particularly (or freshly prepared??) prepared samples (see 2.32). All measurements were made at a relative humidity of $>90 \%$ r.H. in the SKP-chamber (see 2.3). For a better classification of corrosion protection of various pre-treatments, different alkaline cleaned references were chosen - one without any coatings and another one without pre-treatment but with organic coating (clear-coat). Compared to the complete uncoated reference with a Volta potential difference ("potential") dominated by a Zn-oxyhydroxide layer, the potential at the intact area of the clear-coat has been shifted cathodically. This shift of about $250 \mathrm{mV}$ can be explained by the barrier effect of the organic coating [32].

In Fig. 7a the line-scans of the clear-coated reference without pre-treatment are plotted and the electrochemical coupling of the defect with the intact area of the clear-coat is evident. While the potential is determined in the defect area by the anodic zinc dissolution (approximately -700 $\mathrm{mV}_{\mathrm{SHE}}$ ), the cathodic ORR and the surface chemistry (Znoxyhydroxides, organic coating) determine the potential at the delamination front and at the 
intact area respectively (up to $-250 \mathrm{mV}$ SHE). Thus the potential difference between the defect and intact area is approximately $450 \mathrm{mV}$ and the average delamination velocity is $371 \pm 55$ $\mu \mathrm{m} / \mathrm{h}$.

In samples pre-treated with a $\mathrm{Zr}$-based conversion layer $\left(\mathrm{ZrO}_{2}\right)$ the potential at the intact clear-coated area is $-650 \mathrm{mV}_{\text {SHE}}$. Thus the potential difference between defect and intact area is merely 150 and $200 \mathrm{mV}$ and hence up to $300 \mathrm{mV}$ smaller compared to the reference without pre-treatment (Fig. 7b). Also the average delamination velocity is significant lower. Various working groups show that the height of this potential difference determines the delamination velocity decisively [22,32]. This means that the electron transfer reactions at the defect area (zinc dissolution) and at the delamination front (ORR) could be successfully retarded by the electronic insulating properties of the $\mathrm{ZrO}_{2}$-conversion layer.

However, for a targeted optimization of a Zr-based conversion layer, it is essential to identify the critical interface of delamination. As a consequence, the clear coat has been directly removed after the delamination experiments and examined by XPS. In addition, the surface of the delaminated metallic substrate was investigated. The comparison of the $\mathrm{Zr} 3 \mathrm{~d}$ element spectra in Fig. $8 \mathrm{~b}$ shows that the conversion layer remained intact on the metallic substrate. This proves that the delamination of the clear coat occurred at the interface between conversion layer and organic coating. Thus the Zr-based conversion layer does not achieve a stable chemical bonding between the clear coat and the metallic substrate. It is assumed that the bonding between the hydroxyl groups of the conversion layer and the organic coating are only based on weak intermolecular interactions. Hence the electrolyte can penetrate through the organic coating to the conversion layer interface followed by a wet de-adhesion of the organic coating. Similar observation were described by Wapner et al. on modified adhesive/SiOx-interfaces [28,31]. 
1 By modification the conversion layer with an organofunctional silane, the interface is

2 stabilized against wet de-adhesion. Surface analytical investigations clearly showed that

3 sample VBH-S has a defined layer structure with suitable functional groups. The adhesion

4 promoter $\left(\gamma\right.$-GPS) reacts by condensation of silanol functional groups with the $\mathrm{ZrO}_{2}-$

5 conversion layer and by reaction with epoxy and carboxyl functional groups with the organic

6 coating during the cross-linking process. SKP measurements reveal the significant decreased

7 delamination velocity $(16 \pm 10 \mu \mathrm{m} / \mathrm{h}$, Fig. $8 \mathrm{~b})$. Depending on the application procedure the one-step preparation process (sample VBH-E) leads to a non-homogenous layer structure of the applied conversion coating. The competitive reaction between the deposition of the inorganic $\mathrm{ZrO}_{2}$-film and the adsorption process of the $\gamma$-GPS on the metallic substrate results in $\mathrm{ZrO}_{2}$ - and silane-rich domains on the metallic surface. This surface pre-treatment has weak barrier and adhesion properties. Thus the measured delamination velocity is $44 \pm 13 \mu \mathrm{m} / \mathrm{h}$ and therefore higher compared to sample VBH-S. For a better overview all delamination velocities of the different specimens are shown in table 3.

\section{Conclusion}

The influence of bifunctional organosilanes on Zr-based conversion layers on hot dip galvanized steel sheets was studied by various surface analytical methods and scanning Kelvin Probe measurements. Two different preparation methods were compared in order to investigate the formation of the layer structures. In the two step coating procedure the metallic substrate was immersed subsequent in the $\mathrm{H}_{2} \mathrm{ZrF}_{6}$ conversion solution and the $\gamma$-GPS containing solution, whereas only one coating solution with both hexafluorozirconic acid and $\gamma$-GPS was used in the one step coating procedure.

The preparation methods significantly influence the surface layer properties of the different samples as was demonstrated by surface analytical studies. The two step coating procedure reveals a distinct homogenous distribution of the Zr-based conversion layer and the $\gamma$-GPS 
1 compared to the one step coating procedure. This can be explained by the absence of any

2 competitive reaction between the organosilane and the hexafluorozirconic acid with the

3 metallic substrate during immersion in the conversion solution. In contrast, the one step

4 coating procedure leads to primarily $\mathrm{ZrO} 2$ or $\gamma$-GPS coated areas on the surface as could be

5 shown by Raman images and XPS depth profiles.

6 In situ HR-SKP measurements were conducted to study the delamination properties of the

7 different prepared conversion layers after subsequent application of an organic coating. It was

8 clearly shown that the pure inorganic Zr-based conversion layer of the two step procedure

9 decelerate the electron transfer reaction kinetics at the defect area of the organic coating and at its delamination front due to the electronic insulating properties of the $\mathrm{ZrO} 2$-conversion layer. However, the Zr-based conversion layer does not achieve a stable chemical bonding to the organic coating (clear coat). As a consequence, the electrolyte can penetrate easily the interface between the conversion layer and the organic coating and wet de-adhesion of the polymer occurs $(\mathrm{vDel}=44 \pm 13 \mu \mathrm{m} / \mathrm{h}$ ). By modification the conversion layer with a distinct $\gamma$-GPS layer in two step procedure a considerable stabilisation of the interface against wet deadhesion could be achieved (vDel $=16 \pm 10 \mu \mathrm{m} / \mathrm{h})$. 
2 [1] F. Eppensteiner, M. Jenkins, Chromate conversion coatings, Metal Finish (2005) 367.

3 [2] Z. Mekhalif, L. Forget, J. Delhalle, Investigation of the protective action of chromate

4 coatings on hot-dip galvanized steel: role of wetting agents, in: Corrosion, Electrodeposition

5 and Surface treatment of the $54^{\text {th }}$ Annual Meeting of the ISE 47, 2005, p. 547.

6 [3] L. Forget, J. Delhalle, Z. Mekhalif, Application of scanning Kelvin probe to study the

7 corrosion protection of chromated hot-dip galvanized steel, Materials and Corrosion 52 $8 \quad(2001) 181$.

9 [4] T.S.N.S. Sanakara, Surface Pretreatment by Phosphate Conversion Coatings - A Review,

Reviews on Advanced Materials Science 9 (2005) 130.

[5] J. Donofrio, Zinc phosphating, Metal finishing 98 (2000) 57.

[6] Agency for Toxic Substance, U.S. Public Health Service, Toxicological Profile for Chromium, ATDSRyTP-88y10, 1989.

[7] European parliament. Directive 2000/53/eg.

[8] European parliament. Directive 2002/95/eg.

[9] H. Li, K. Liang, L. Mei, S. Gu, S. Wang, Corrosion protection of mild steel by zirconia sol-gel coatings, Journal of Materials Science Letters 20 (2001) 1081.

[10] G. Gusmano, G. Montesperelli, M. Rapone, G. Padeletti, A. Cusmà, S. Kaciulis, A.Mezzi, R. Di Maggio, Zirconia primers for corrosion resistant coatings, Surface and Coatings Technology 201 (2007) 5822. 
1 [11] R. Di Maggio, L. Fedrizzi, S. Rossi, Effect of the chemical modification of the precursor

2 of $\mathrm{ZrO}_{2}$ films on the adhesion of organic coatings, Journal of Adhesion Science and

3 Technology $15(2001) 793$.

4 [12] L. Fedrizzi, F.J. Rodriguez, S. Rossi, F. Deflorian, R. Di Maggio, The use of elec5 trochemical techniques to study the corrosion behaviour of organic coatings on steel 6 pretreated with sol-gel zirconia films, Electrochimica Acta 46 (2001)3715.

7 [13] O. Lunder, F. Lapique, B. Johnsen, K. Nisancioglu, Effect of pre-treatment on the durability of epoxy-bonded AA6060 aluminium joints, International Journal of Adhesion and $9 \quad$ Adhesives $24(2004) 107$.

[14] O. Lunder, C. Simensen, Y. Yu, K. Nisancioglu, Formation and characterisation of Ti-Zr based conversion layers on AA6060 aluminium, Surface and Coatings Technology 184 (2004) 278.

[15] J.H. Nordlien, J.C. Walmsley, H. Østerberg, K. Nisancioglu, Formation of a zirconiumtitanium based conversion layer on AA 6060 aluminium, Surface and Coatings Technology $153(2002) 72$.

[16] P. Laha, T. Schram, H. Terryn, Use of spectroscopic ellipsometry to study Zr/Ti films on

Al, Surf. Interface Anal. 34 (2002) 677.

[17] D. Chidambaram, C.R. Clayton, G.P. Halada, The role of hexafluorozirconate in the formation of chromate conversion coatings on aluminum alloys, Electrochimica Acta 51 (2006) 2862.

[18] F. Andreatta, A. Turco, I. de Graeve, H. Terryn, J.H.W. de Wit, L. Fedrizzi, SKPFMand

SEM study of the deposition mechanism of $\mathrm{Zr} / \mathrm{Ti}$ based pre-treatment on AA6016 aluminum alloy, Surface and Coatings Technology 201 (2007)7668. 
1 [19] P. Puomi, H.M. Fagerholm, J.B. Rosenholm, K. Jyrkäs, Comparison of different

2 commercial pretreatment methods for hot-dip galvanized and Galfan coated steel, Surface and

3 Coatings Technology 115 (1999) 70.

4 [20] P. Puomi, H.M. Fagerholm, J.B. Rosenholm, R. Sipilä, Optimization of commercial 5 zirconic acid based pretreatment on hot-dip galvanized and Galfan coated steel, Surface and $6 \quad$ Coatings Technology 115 (1999) 79.

7 [21] S. Verdier, N. van der Laak, F. Dalard, J. Metson, S. Delalande, An electrochemical and 8 SEM study of the mechanism of formation, morphology, and composition of titanium or 9 zirconium fluoride-based coatings, Surface and Coatings Technology 200 (2006) 2955.

[22] C. Stromberg, P. Thissen, I. Klueppel, N. Fink, G. Grundmeier, Synthesis and characterisation of surface gradient thin conversion films on zinc coated steel, Electrochimica Acta 52 (2006) 804.

[23] S. Adhikari, K.A., Unocic, Y., Zhai, G.S., Frankel, J., Zimmerman, W. Fristad, Hexafluorozirconic acid based surface pretreatments: Characterization and performance assessment, Advances in corrosion science for lifetime prediction and sustainability Selection of papers from the 8th ISE Spring Meeting 2-5May 2010, Columbus, OH, USA 56 (2011) 1912.

[24] F.O. George, P. Skeldon, G.E. Thompson, Formation of zirconium-based con-version coatings on aluminium and Al-Cu alloys, Corrosion Science 65 (2012) 231.

[25] T. Lostak, S. Krebs, A. Maljusch, T. Gothe, M. Giza, M. Kimpel, J. Flock, S. Schulz, Formation and characterization of $\mathrm{Fe}^{3+}-/ \mathrm{Cu}^{2+}$-modified zirconium oxide conversion layers on zinc alloy coated steel sheets, Electrochimica Acta 112 (2013) 14. 
1 [26] T. Lostak, A. Maljusch, B. Klink, S. Krebs, M. Kimpel, J. Flock, S. Schulz, W.

2 Schuhmann, Zr-based conversion layer on Zn-Al-Mg alloy coated steel sheets: insights into

3 the formation mechanism, Electrochimica Acta 137 (2014) 65.

4 [27] P. Taheri, K. Lill, J.H.W. de Wit, J.M.C. Mol, H. Terryn, Effects of Zinc Surface Acid-

5 Based Properties on Formation Mechanisms and Interfacial Bonding Properties of Zirconium-

6 Based Conversion Layers, J. Phys. Chem. C 116 (2012) 8426.

7 [28] K. Wapner, G. Grundmeier, Scanning Kelvin probe measurements of the stability of 8 adhesive/metal interfaces in corrosive environments, Advanced Engineering Materials 6 9 (2004) 163.

[29] B. Schinkinger, R. Petzold, H.-J. Tiller, G. Grundmeier, Chemical structure and morphology of ultrathin combustion CVD layers on zinc coated steel, Applied Surface Science 179 (2001) 79 .

[30] B. Schinkinger, Schichtanalytische und elektrochemische Untersuchungen zur Abscheidung dünner SiO2- und Organosilanschichten auf verzinktem Stahl, Ph.D. thesis, Bochum (2004).

[31] K. Wapner, Grenzflächenchemische und elektrochemische Untersuchung zur Haftung und Enthaftung an modifizierten Klebstoff/Metall-Grenzflächen, Ph.D. thesis, Bochum (2006).

[32] G. Klimow, N. Fink, G. Grundmeier, Eletrochemical studies of the inhibition of organically coated galvanised steel by thin conversion films, Electrochimica Acta 53 (2007) 1290.

[33] A. Collazo, A. Covelo, C. Perez, Structural transformation of silane-based zirconiummodified sol-gel-coatings, Surf. and Inter. Anal. 42 (2010) 1201. 
1 [34] Y. Ivanova, T. S. Gerganova, Y. Dimiriev, I. M. Miranda Salvado, M. H. V. Fernandes,

2 Nanostructured hybrid materials as precursors for synthesis of nanocomposites in Si-O-C-N-

3 Zr system. INTERNATIONAL CONFERENCE ON SURFACES, COATINGS AND

$4 \quad$ NANOSTRUCTURED MATERIALS nanoSMat 515 (2006) 271.

5 [35] M. Hesse, H. Meier, B. Zeeh, Spektroskopische Methoden in der organischen Chemie, 6 Georg Thieme Verlag, Stuttgart (1991).

7 [36] D. Lin-Vien, N. Colthup, W. Fateley, J. Grasselli, The Handbook of Infrared and Raman

8 Characteristic Frequencies of Organic Molecules, Academic Press Inc., San Diego (1991).

9 [37] C. Rau, W. Kulisch, Mechanism of plasma polymerization of various silicon organic monomers, Thin Solid Films, 249 (1994) 28.

[38] J. F. Moulder, W. F. Stickle, P.E. Sobol, K. D. Bomben, Handbook of X-Ray Photoelectron Spectroscopy, Physical Electronics, Inc. Minnesota (1995).

[39] H. Ardelean, I. Frateur, P. Marcus, Corrosion protection of magnesium alloys bycerium, zirconium and niobium-based conversion coatings, Corrosion Science50 (2008) (1907).

[40] W. Bensch, O. Helmer, M. Muhler, H. Ebert, M. Knecht, Experimental and the-oretical bandstructure of the layer compound ZrSiTe, The Journal of PhysicalChemistry 99 (1995) 3326.

[41] Y.M. Wang, Y.S. Li, P.C. Wong, K.A.R. Mitchell, XPS studies of the stability andreactivity of thin films of oxidized zirconium, Applied Surface Science 72 (1993) 237.

[42] S. K. Tan, K. L. Yeo, A. T. S. Wee, In situ XPS and SIMS analysis of O2+ beaminduced silicon oxidation, Surf. and Inter. Anal., 36 (2004) 640.

[43] G. Beamson, D. Briggs, High Resolution XPS of Organic Polymers: the Scienta ESCA Database, Wiley, Chichester (1992). 
1 [44] C. G. Pantano, T. N. Wittberg, XPS analysis of silane coupling agents and silane-treated 2 e-glass fibers, Surf. and Inter. Anal., 15 (1990) 498.

3 [45] G. Grundmeier, P. Thiemann, J. Carpentier, N. Shirtcliffe, M. Stratmann, Tailoring of the 4 morphology and chemical composition of thin organosilane microwave plasma polymer layers on 5 metal substrates. Thin Solid Films, 446(1):61-71, 2004

6 [46] V. G. Keramidas, W. B. White, Raman scattering study of the crystallization and phase 7 transformations of $\mathrm{ZrO}_{2}$, Journal of the American Ceramic Society, 57(1):22-24, 974

8 [47] T. Dieing, O. Hollricher, J. Toporski, Confocal Raman Microscopy, 1st Edition, Springer-Verlag $9 \quad$ Berlin Heidelberg, 2011 
2 Table 1: Composition of the different conversion solutions and the used application 3 parameters.

4 Table 2: Concentration of chemical elements present on the surface of the conversion layer as 5 determined by XPS.

6 Table 3: Delamination velocities of different conversion coatings on coated specimens.

\section{$7 \quad$ Figure captions}

8 Figure 1: Representation of XPS survey spectra (VBH-E, VBH-S).

9 Figure 2: Single element XPS depth profile (VBH-S). The Si2p-signal was increased 10 times. Reference sputter rate: $\mathrm{R}\left(\mathrm{SiO}_{2}\right)=8 \mathrm{~nm} / \mathrm{min}$.

Figure 3: Single element XPS depth profile (VBH-E). Reference sputter rate: $\mathrm{R}(\mathrm{SiO} 2)=8 \mathrm{~nm} / \mathrm{min}$.

Figure 4: IRRA spectra obtained from sample VBH-S and VBH-E in the range of 4000$14250 \mathrm{~cm}^{-1}$.

Figure 5: Raman spectra obtained from sample VBH-S and VBH-E in the range of 4000$250 \mathrm{~cm}^{-1}$.

Figure 6: Optical images obtained through the Raman microscope from sample VBH-S (left) and VBH-E (right) after deposition of $\mathrm{ZrO}_{2}$ and $\gamma$-GPS on the surface. The white rectangles indicate the Raman imaging area carried out on the surfaces. The Raman images show the distribution of $\mathrm{ZrO}_{2}$ (used vibration area at $550-350 \mathrm{~cm}^{-1}$, green) and $\gamma-\mathrm{GPS}\left(3000-2800 \mathrm{~cm}^{-}\right.$ $21 \quad$; red). 
1 Figure 7: HR-SKP line scans of clear coated samples during the cathodic delamination

2 process under high humidity. a) uncoated, alkaline cleaned reference; b) ZrO2 conversion 3 layer; c) VBH-S; d) VBH-E.

4 Figure 8: a) Comparison of XPS survey spectra of various interfaces (metallic substrate, top 5 and underside of the delaminated clear coat); b) comparison of the XPS Zr element spectra of 6 various interfaces (metallic substrate, top and underside of the delaminated clear coat). 
Figure 1

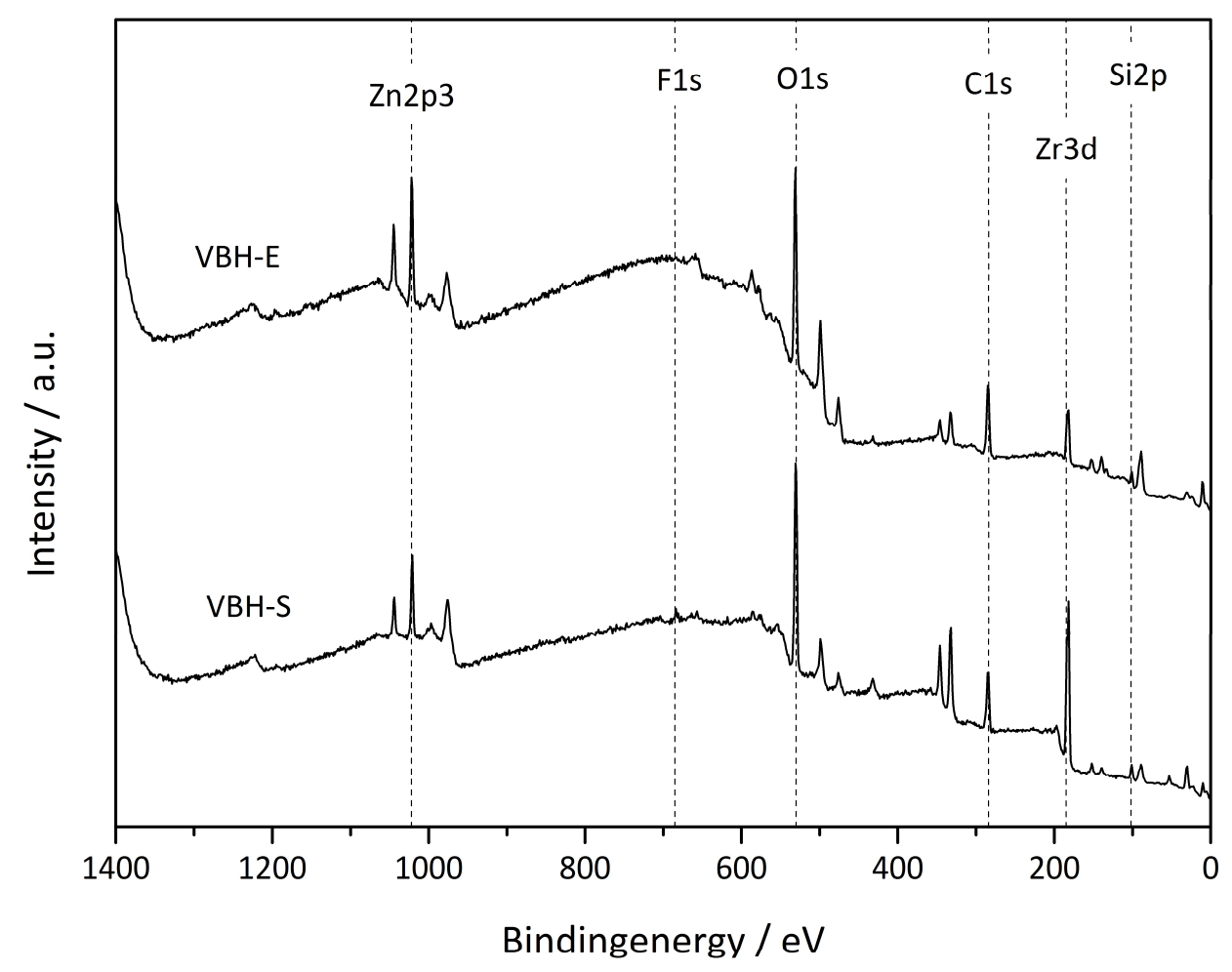

3 
Figure 2

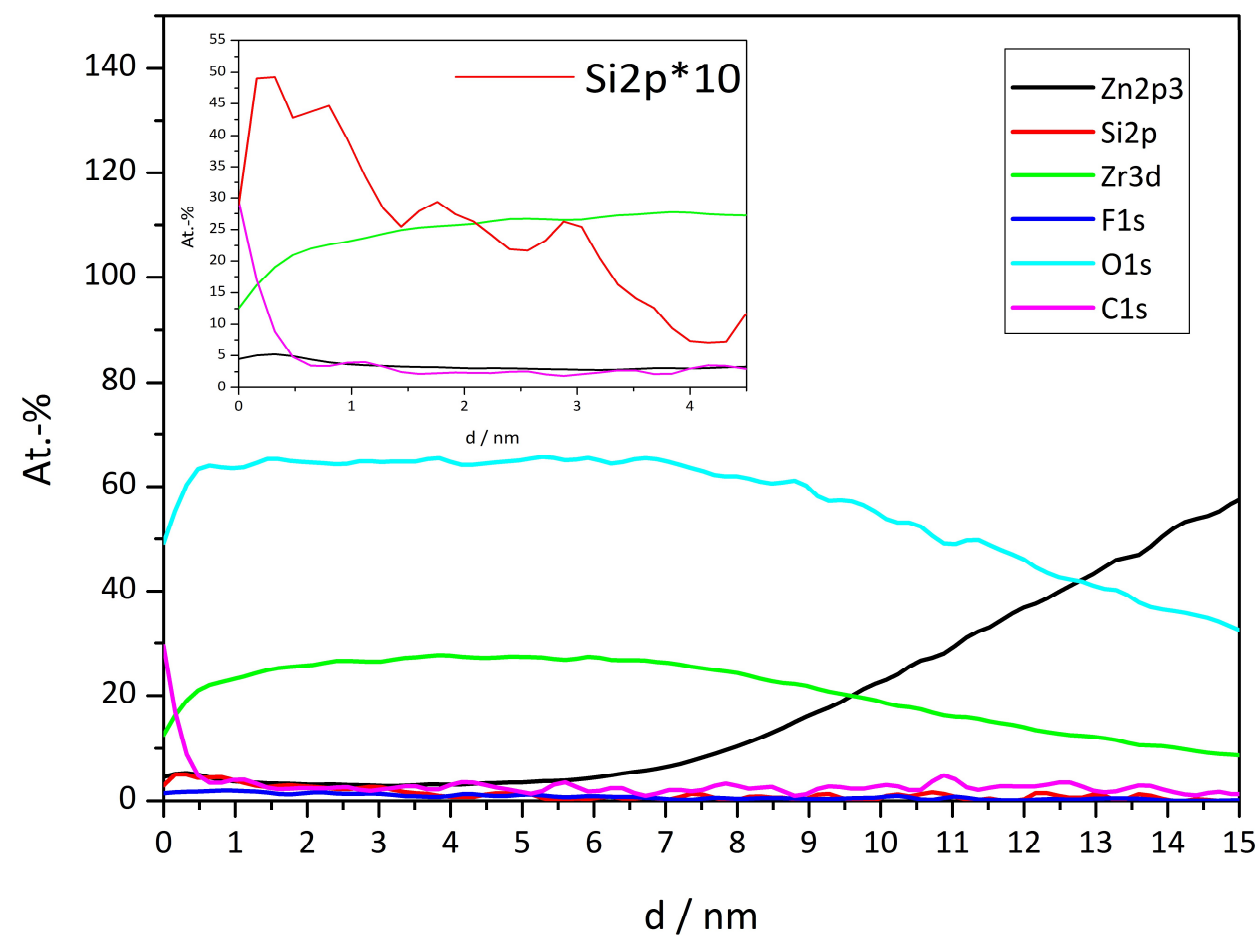

2

3 
Figure 3

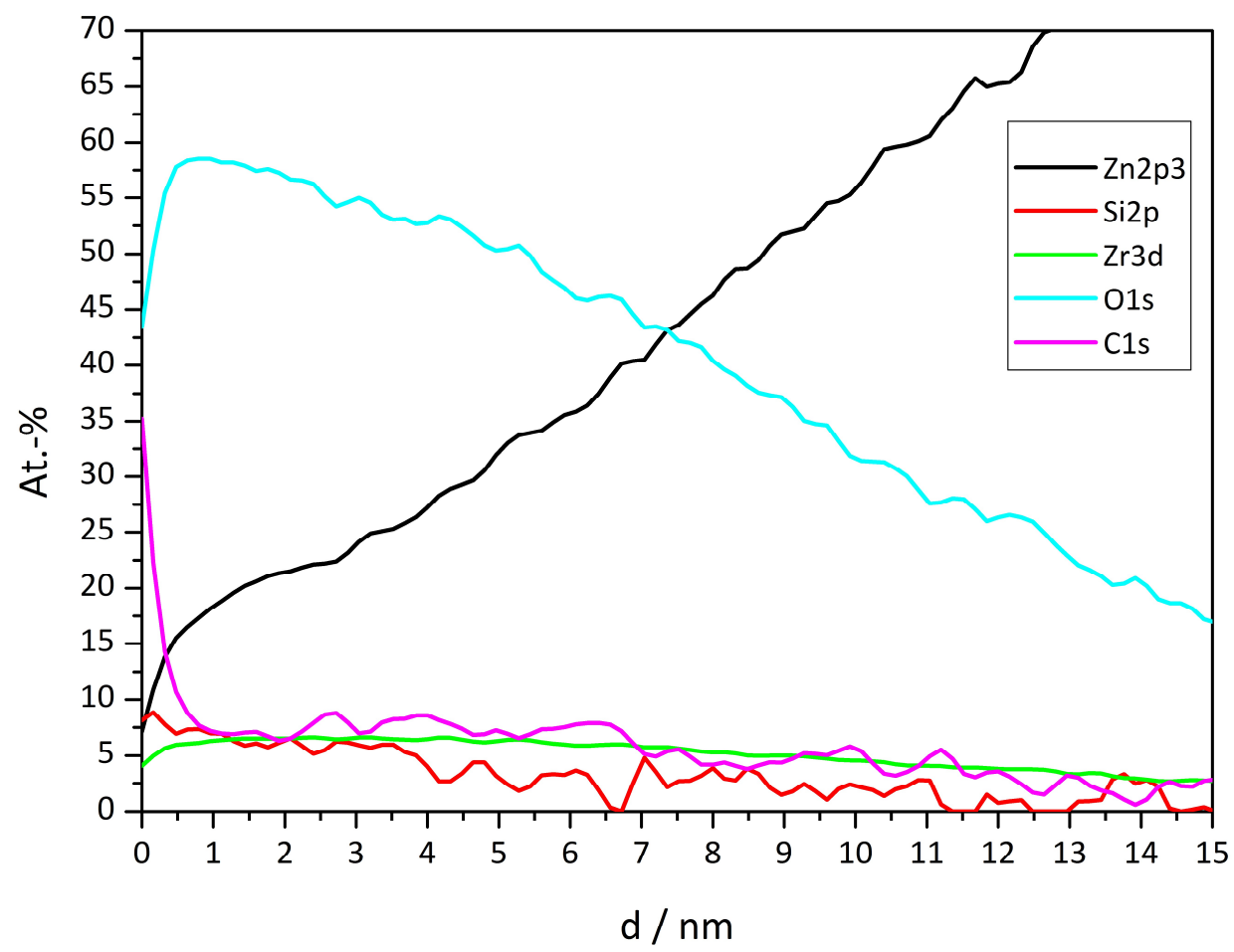

2

3 
Figure 4

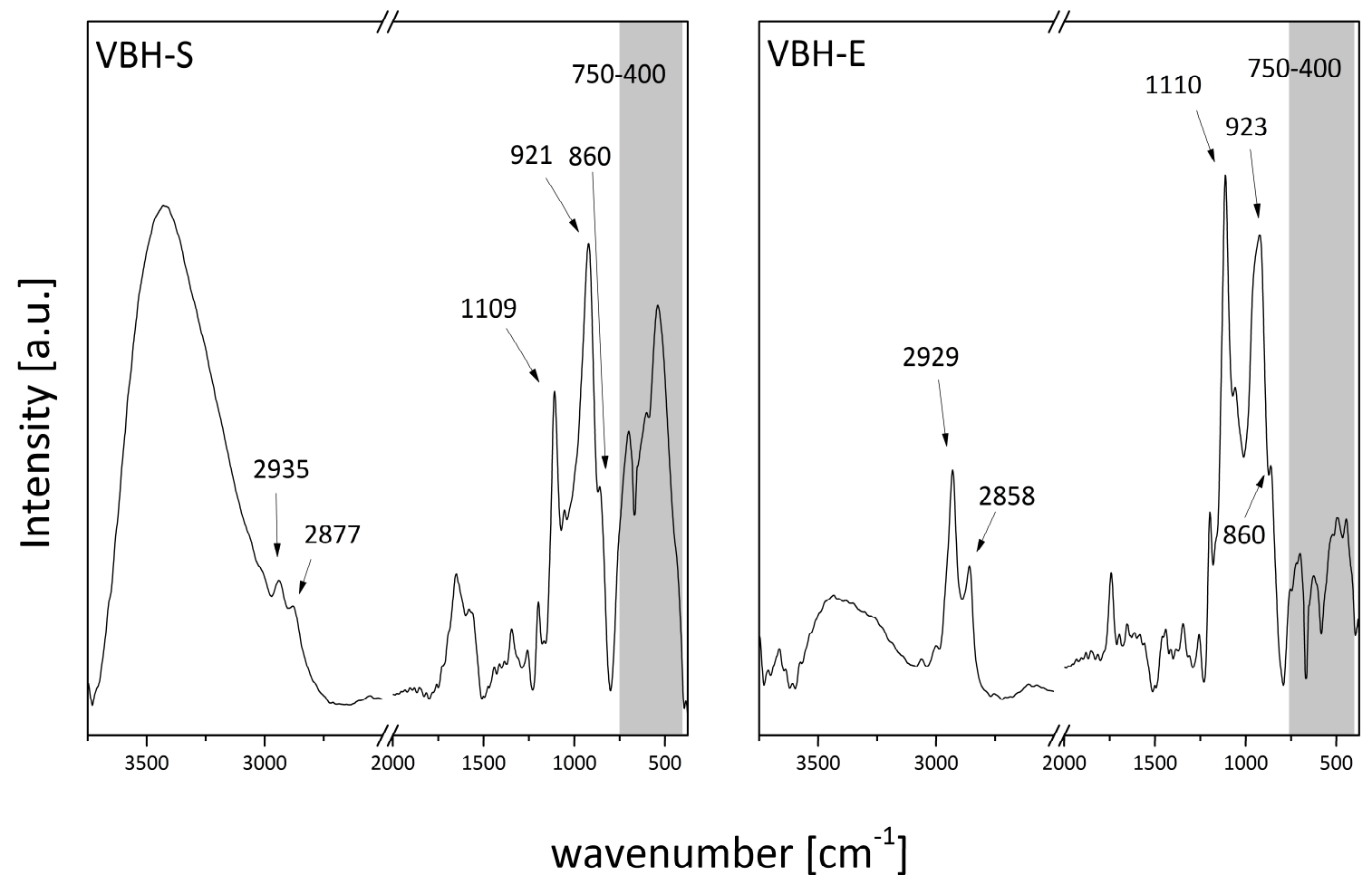

2

3 
Figure 5

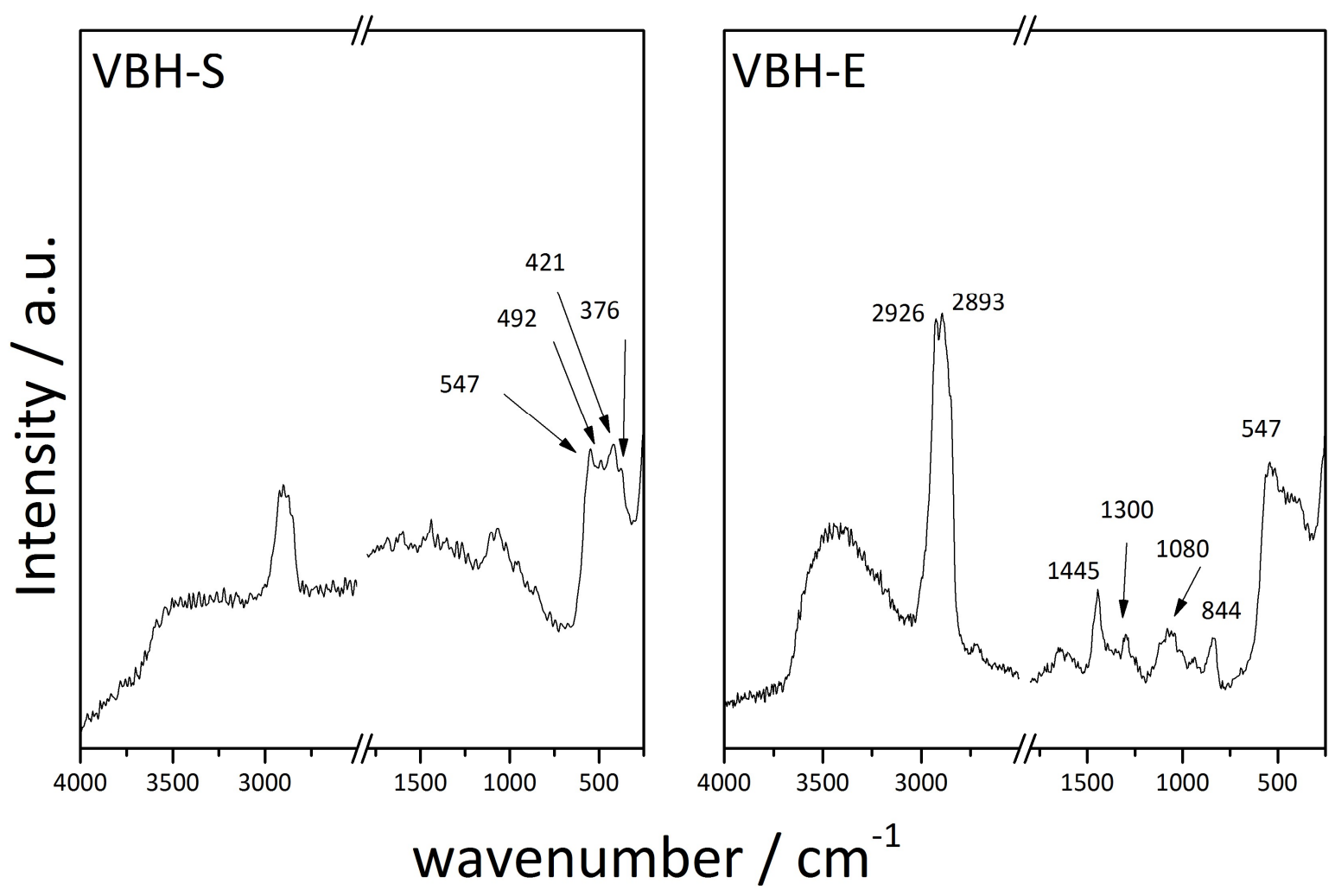

3 

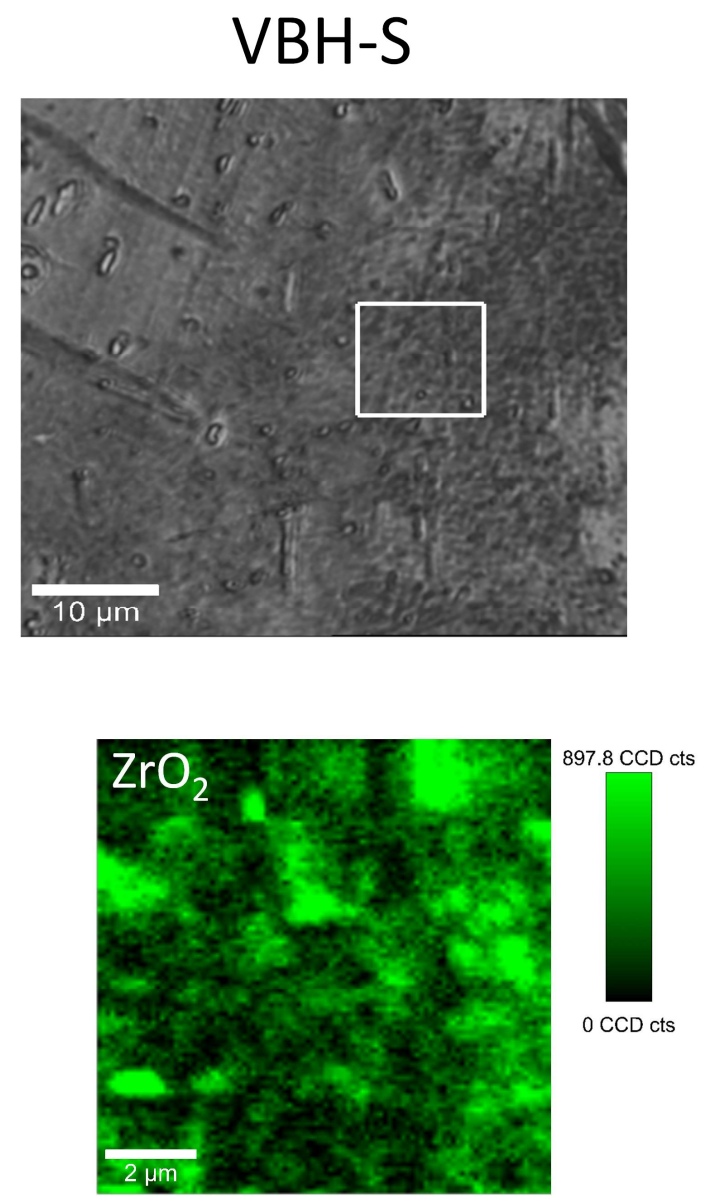

2
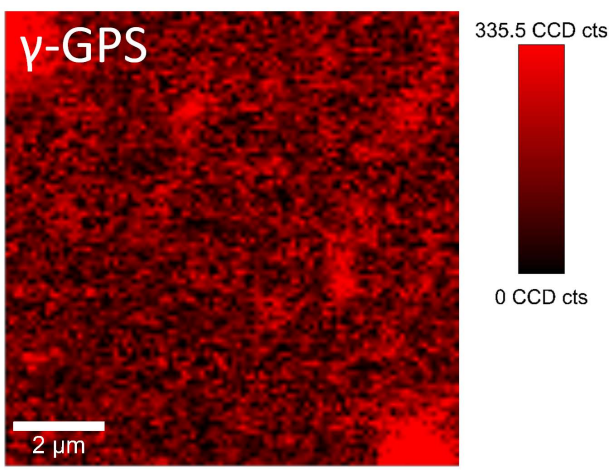

VBH-E
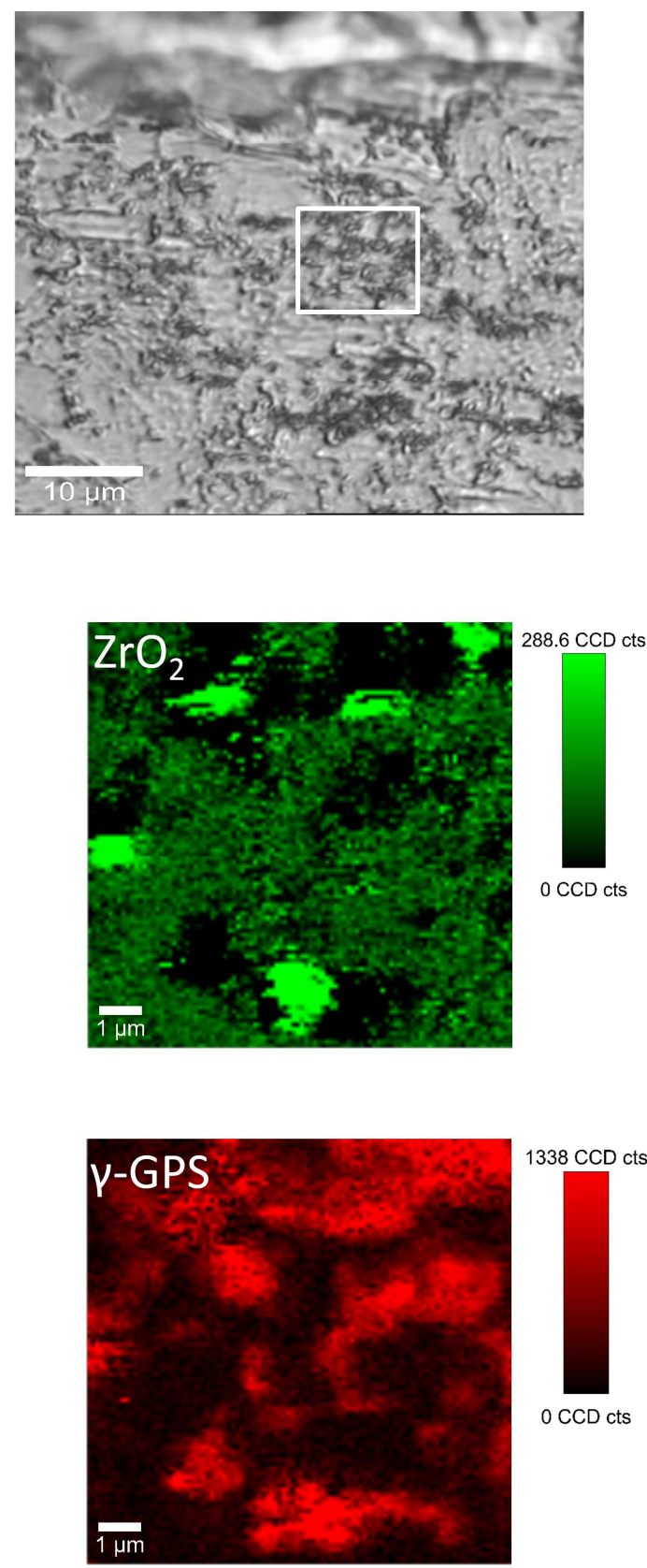
Figure 7
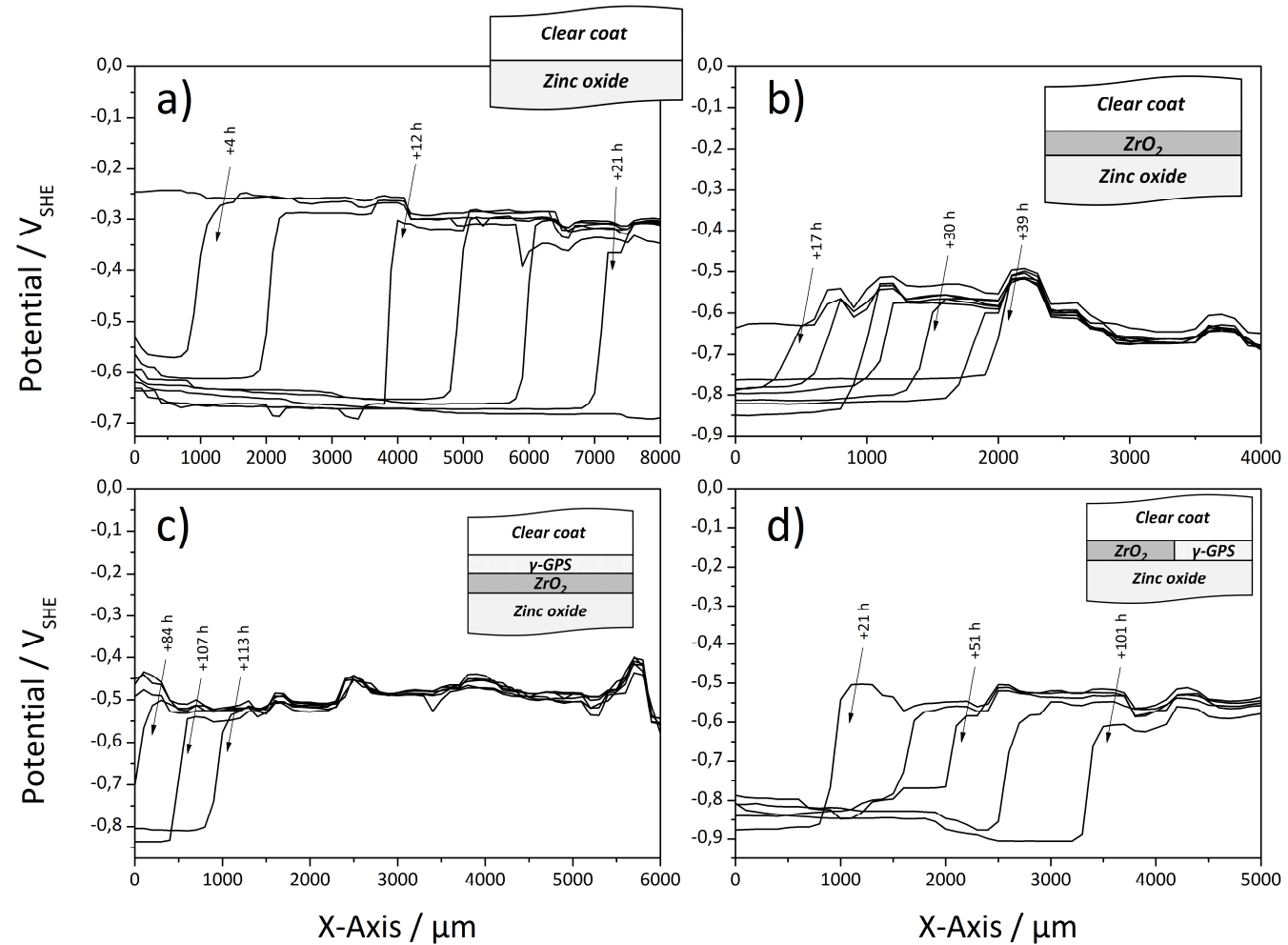

2

3 
Figure 8
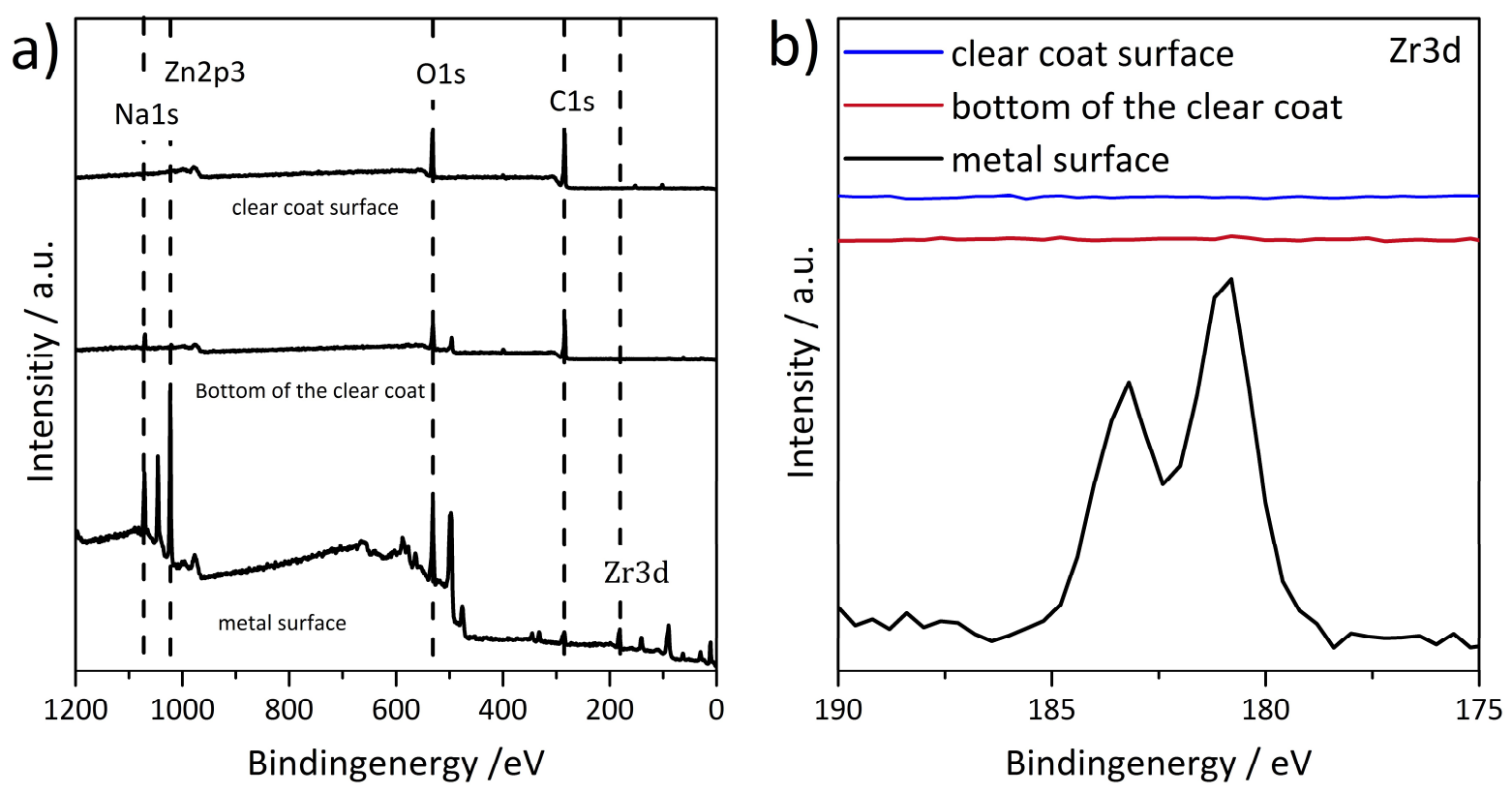
Table 1

2

\begin{tabular}{|c|c|c|c|}
\hline Sample & Conversion solution & pH & Treatment time \\
\hline $\mathrm{ZrO}_{2}$ & $1 \mathrm{mmol} / 1 \mathrm{H}_{2} \mathrm{ZrF}_{6}$ & 4 & 90 \\
\hline VBH-E & $1 \mathrm{mmol} / 1 \mathrm{H}_{2} \mathrm{ZrF}_{6}+1$ wt. $\% \gamma$-GPS & 4 & 90 \\
\hline VBH-S & $\begin{array}{l}\text { Step a) } 1 \mathrm{mmol} / 1 \mathrm{H}_{2} \mathrm{ZrF}_{6} \\
\text { Step b) } 1 \mathrm{wt} . \% \gamma \text {-GPS }\end{array}$ & 4 & 90 \\
\hline
\end{tabular}

3 
1

2

\begin{tabular}{|c|c|c|c|c|c|c|}
\hline \multirow{2}{*}{ Sample } & \multicolumn{6}{|c|}{ Element / At.\% } \\
\cline { 2 - 7 } & Zn2p3 & Si2p & Zr3d & F1s & O1s & C1s \\
\hline VBH-S & 5 & 5 & 16 & 2 & 55 & 17 \\
\hline VBH-E & 11 & 9 & 5 & - & 53 & 22 \\
\hline
\end{tabular}

3 
Table 3

2

\begin{tabular}{|l|l|}
\hline Sample & Delamination velocity / $\boldsymbol{\mu} \mathbf{m ~ h}^{\mathbf{1}}$ \\
\hline Uncoated, alkaline cleaned $\mathrm{ZnO}-$ Reference & $371 \pm 55$ \\
\hline $\mathrm{ZrO}_{2}$ & $76 \pm 9$ \\
\hline $\mathrm{VBH}-\mathrm{S}$ & $16 \pm 10$ \\
\hline VBH-E & $44 \pm 13$ \\
\hline
\end{tabular}

3 
DuEPublico

Duisburg-Essen Publications online

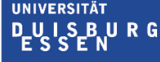

offen im Denken

Ub $\mid \begin{aligned} & \text { universitäts } \\ & \text { bibliothek }\end{aligned}$

This text is made available via DuEPublico, the institutional repository of the University of Duisburg-Essen. This version may eventually differ from another version distributed by a commercial publisher.

DOI: $\quad$ 10.1016/j.surfcoat.2016.08.030

URN: urn:nbn:de:hbz:464-20201204-123833-9

This is the Authors Accepted Manuscript of an article published in: Surface and Coatings Technology 305 (2016) 223-230. The final version may be found at: https://doi.org/10.1016/j.surfcoat.2016.08.030

This work may be used under a Creative Commons Attribution -

NonCommercial - NoDerivatives 4.0 License (CC BY-NC-ND 4.0) 